\title{
Hue discrimination in peripheral vision under conditions of dark and light adaptation*
}

\author{
BRUCE A. AMBLER $\dagger$ \\ University of Oregon, Eugene, Oregon 97403
}

\begin{abstract}
Rod interference is a possible factor contributing to the elevation of chromatic threshold in peripheral vision. It was found that light adaptation lowered peripheral chromatic thresholds. This result was interpreted as being due to the lowering of rod sensitivity. It was also found that light in the photochromatic interval appeared blue, indicating that rods may add a blue component to peripheral color vision.
\end{abstract}

The ability to discriminate color is degraded in peripheral vision. Ferree and Rand (1919) and Spillmann and Seneff (1971) have reported a rise in chromatic (color) thresholds as stimuli are presented further out in the periphery. There are at least two possible factors that could be involved in this effect. One is the decreasing cone density in the periphery (Cornsweet, 1970). However, this hypothesis is not completely satisfactory, given data such as those of Ferree and Rand (1919). They found that in peripheral vision some colors, for example greens, were degraded more than other colors. This finding could not be accounted for by a general reduction of all cone types. Another possible factor contributing to this change in chromatic threshold is the increase in the ratio of the number of rods to the number of cones that occurs in the periphery of the retina. Counter to a simple form of the duplicity theory of vision, it could be proposed that rods interact with cones and degrade the color signal of the cone system. This interaction could occur in at least two different ways. The rods might add an achromatic signal that would desaturate the perceived hue and/or they might add a signal that becomes part of the information for hue used by the color processing system. Due to the broad spectral response of rods, this latter alternative would also distort peripheral color perception. It would cause particular distortion for middle wavelength lights to which rods are most sensitive. The effect of rods on the cone system would be more severe as the rod-to-cone ratio increased in peripheral vision.

Electrophysiological recording has demonstrated a possible mechanism for rod-cone interaction. Ganglion cells receiving input from both rods and cones have been found in the frog (Donner \& Rushton, 1969), cat (Barlow \& Levick, 1967), and monkey (Gouras \& Link, 1966).

Evidence for the influence of rods on peripheral color

*This research, which partially fulfilled the requirements for the PhD degree at the University of Oregon, was supported by NSF Grant GB-24884 aw arded to Professor Jacob Beck. I would like to thank Professor Beck for his many helpful comments and suggestions.

+Now at Department of Psychology, University of Texas at Arlington, Arlington, Texas 76109. vision has been found by holding peripheral position constant and manipulating relative rod-cone sensitivity through adaptation. This manipulation has been based on data like those of Hecht et al (1937), which demonstrated a brief period of about 3-8 min after light adaptation during which the sensitivity of cones relative to that of rods is maximized. During this time, any interference with color perception due to rods should be minimized. As dark adaptation proceeds, rods increase their sensitivity relative to the sensitivity of cones. The increase in sensitivity of rods relative to cones continues for at least $1 / 2 \mathrm{~h}$. As the sensitivity of rods relative to cones increases, the effect of rods on color processing should become stronger.

The procedure has been used by Lie (1963) to demonstrate that rods do play a role in peripheral color vision. Lie light-adapted his Ss and then measured both chromatic and achromatic (seeing light) threshold in the periphery as dark adaptation proceeded. He found that as time in dark increased, not only did the achromatic threshold decrease, but the chromatic threshold increased. This indicated that as rods became more sensitive, peripheral color vision was degraded. Recently, Spillmann and Conlon (1972) performed the same experiment with similar results. However, the increase in chromatic threshold measured $6 \mathrm{deg}$ from the fovea, began after only $3 \mathrm{~min}$ in the dark. The rod-cone break occurred in the achromatic threshold at $8 \mathrm{~min}$ in the dark. The rod-cone break is a change in the slope of achromatic threshold plotted as a function of time in the dark. It is thought to mark the time when achromatic threshold changes from being determined by cones to being determined by rods. If this increase in chromatic threshold is thought to be due to rods, then it would have to be assumed that rods could be increasing their sensitivity relative to cones enough to influence color vision before the rods become sensitive enough to determine achromatic threshold. Spillmann and Conlon are in agreement with $\mathrm{Lie}$ in their finding that as dark adaptation proceeds, chromatic threshold increases.

Lie proposed that rods add an achromatic component that desaturates and therefore reduces the discriminability of the color. This model is consistent 
with his observation that within the photochromatic interval "the light is perceived to change very gradually from a dirty gray-brown color to a constantly purer gray-white color until a faint tint of the hue in question appears." This observation, however, is in marked contrast to several earlier reports.

Roaf (1933), citing his own observations and those of Von Kries, reports that dim light to the dark-adapted eye is a "monochromatic bluish white." Roaf discusses the possibility that rods serve as receptors for blue vision. More recently, Hunt (1952) has reported that the sensation of a dim light presented to the dark-adapted eye is a pale blue and not colorless. Trezona (1970) reviews evidence suggesting that rods do contribute a blue response to the color process. These observations suggest the possibility that rods affect peripheral color vision by contributing an achromatic signal, or a chromatic (blue) signal, or both.

It should be interesting to know whether Lie's failure to see blue in the achromatic interval was due to a failure to imagine a color that was not there or to a failure to observe a sensation that was present. His experimental procedure suggests a possible reason for the latter alternative. While making observations, Lie's Ss were told in advance which stimulus would be presented. This procedure might encourage Ss to set too high a criterion for color sensations that did not fit their expectations and a corresponding lower criterion for those that did. To overcome this difficulty, Ss in the present experiment were not told which color to expect. Chromatic thresholds were measured under conditions of light and dark adaptation. At the same time, a record was kept of what color sensations were reported throughout the photochromatic interval (the interval between achromatic and chromatic threshold) when Ss were not biased toward seeing any one color more than another.

\section{METHOD}

\section{Apparatus}

Four "monochromatic" stimuli were obtained $(470,510,580$, and $670 \mathrm{~nm}$ ) by passing light from a tungsten source through four Bausch and Lomb second-order interference filters. The maximum photopic luminance of the four stimuli was $3.3,21$, $44,2.4 \mathrm{fL}$, respectively, Luminance measurements were made of the stimuli as viewed by the Ss with a Spectra Pritchard light photometer. Exposure duration was maintained at $150 \mathrm{~m} \mathrm{sec}$ $( \pm 5 \mathrm{msec})$ by passing the light beam through a mechanical shutter. Intensity was controlled by an annular neutral density wedge. Light was transmitted to presentation position through a circular bundle of fiber light guides that could be clipped on any position along a perimeter up to $90 \mathrm{deg}$ from the fixation point. The stimulus subtended a visual angle of $56 \mathrm{~min}$ of arc at all presentation positions and was always $11.75 \mathrm{in}$. from S's right eye. The fixation point subtended $19 \mathrm{~m}$ in of arc and was $.072 \mathrm{fL}$

Light adaptation was accomplished by exposing the right eye to three $500-\mathrm{W}\left(3400^{\circ} \mathrm{K}\right)$ photoflood lights. The lights were viewed through a white glass diffusing screen and two Wratten color-correcting filters $(\mathrm{CC} 30 \mathrm{~B}, \mathrm{CC} 05 \mathrm{~B})$. The visual angle of the diffusing screen was $160 \mathrm{deg}$. The filters, surrounded by a black cardboard border, were placed directly in front of the S's eye. Ss fixated the left portion of the diffusing screen, thus presenting the adapting stimulus to the entire right half of the visual field where test stimuli would be presented. The color-correcting filters reduced the amount of light in the long wavelength end of the spectrum relative to the shorter wavelengths. With the use of these filters, Ss reported that there were no colored afterimages after adaptation. Luminance measured through the glass and filters was $585 \mathrm{fL}$. The adaptation period and intertrial interval were measured with two separate electric timers. The minimum intertrial interval was $15 \mathrm{sec}$.

\section{Subjects}

Three university undergraduates were paid $\$ 1.50 / \mathrm{h}$ for participation. Two of the Ss, D.S. and S.G., were naive as to the purpose of the experiment. They knew only that they were reporting observed colors in peripheral positions. S A.S. knew that the experiment involved the role of rods in peripheral color vision, but had no idea that any color might be expected in the photochromatic interval.

Preliminary experiments were run on all Ss with foveal stimulus presentation. Chromatic thresholds were obtained with the double-staircase method (Cornsweet, 1962). This gave Ss practice at making color judgments and tested them for normal foveal color sensitivity.

The thresholds were compared to the data of Wald (1945). Since the relative sensitivity to the four wavelengths was of concern, the shape of the Wald foveal spectral sensitivity function was compared to that of the three Ss. The shape of the Ss' functions was the same as the function presented by Wald. The data presented no evidence for abnormal color vision.

\section{Procedure}

The general plan of the experiment was to obtain both chromatic and achromatic thresholds under two different conditions of adaptation. In the light-adaptation condition, the right eye was exposed to the adapting light for $2 \frac{1}{2} \mathrm{~min}$. Threshold measurements were obtained during a period beginning at $3 \mathrm{~min}$ and ending at $6 \mathrm{~min}$ af ter the adapting light was turned off. During this period, cone sensitivity is nearly maximized, while rod sensitivity is still degraded. In the dark-adaptation condition, after $1 / 2 h$ of dark adaptation, rod sensitivity was maximized and the influence of rods on chromatic thresholds and color sensations in the interval between achromatic and chromatic threshold should be observed.

Both chromatic and achromatic thresholds were obtained using an increasing method of limits. Luminance was doubled on each successive trial. Ss were presented randomly with five different stimuli; one achromatic and four monochromatic stimuli. The maximum luminance of the achromatic stimulus was $6.7 \mathrm{fL}$. Ss were allowed to respond that the light appeared red, green, blue, yellow, achromatic, or that they had seen nothing. Care was taken that the S's responses would not be biased. All five stimuli were presented equally often, and the $S$ did not know which stimulus was being presented. When responses were given, the $E$ recorded them without reaction. Ss were also told that $20 \%$ of the trials would be a colorless stimulus. This was designed to inhibit guessing. It was determined that a chromatic threshold had been reached when Ss reported the correct hue and maintained that report for two more increments in a row. That is, the threshold was taken as the first appropriate report in a series of three appropriate reports in a row. Achromatic threshold was taken at the first of three consistent reports that light had been seen. Stimuli were presented at 8 and $72 \mathrm{deg}$ in the right visual field, first under dark adaptation and then under light adaptation. A total of nine ascending series per $S$ was presented for each stimulus at each peripheral position. The stimuli were viewed through the natural pupil. 
Table 1

Peripheral Chromatic Thresholds: Mean Photopic Luminance (fL)

\begin{tabular}{|c|c|c|c|}
\hline & \multirow[b]{2}{*}{$\mathbf{S}$} & \multicolumn{2}{|c|}{ Eccentricity of Stimulus } \\
\hline & & $\begin{array}{c}\text { Light } \\
\text { Adapted }\end{array}$ & $\begin{array}{c}\text { Dark } \\
\text { Adapted }\end{array}$ \\
\hline & & \multicolumn{2}{|c|}{ 470-nm Stimulus } \\
\hline $8 \mathrm{Deg}$ & $\begin{array}{l}\text { A.S. } \\
\text { D.S. } \\
\text { S.G. }\end{array}$ & $\begin{array}{l}.00095 \\
.00047 \\
.049\end{array}$ & $\begin{array}{l}.00014 \\
.013 \\
.0080\end{array}$ \\
\hline $72 \mathrm{Deg}$ & $\begin{array}{l}\text { A.S. } \\
\text { D.S. } \\
\text { S.G. }\end{array}$ & $\begin{array}{l}.0024 \\
.008 \\
.19\end{array}$ & $\begin{array}{l}* \\
* \\
*\end{array}$ \\
\hline 8 Deg & $\begin{array}{l}\text { A.S. } \\
\text { D.S. } \\
\text { S.G. }\end{array}$ & $\begin{array}{l}.0037^{5} \\
.0015 \\
.0070\end{array}$ & $\begin{array}{l}\text { lus } \\
.025 \\
.033 \\
.075\end{array}$ \\
\hline $72 \mathrm{Deg}$ & $\begin{array}{l}\text { A.S. } \\
\text { D.S. } \\
\text { S.G. }\end{array}$ & $\begin{array}{r}.56 \\
.24 \\
1.90\end{array}$ & $\begin{array}{l}* \\
* \\
*\end{array}$ \\
\hline $8 \mathrm{Deg}$ & $\begin{array}{l}\text { A.S. } \\
\text { D.S. } \\
\text { S.G. }\end{array}$ & $\begin{array}{l}.0044 \\
.0023 \\
.0145\end{array}$ & $\begin{array}{l}\text { lus } \\
\qquad .032 \\
.0044 \\
.145\end{array}$ \\
\hline 72 Deg & $\begin{array}{l}\text { A.S. } \\
\text { D.S. } \\
\text { S.G. }\end{array}$ & $\begin{array}{l}.36 \\
.16 \\
*\end{array}$ & $\begin{array}{l}* \\
* \\
*\end{array}$ \\
\hline & & \multicolumn{2}{|c|}{$670-\mathrm{nm}$ Stimulus } \\
\hline $8 \mathrm{Deg}$ & $\begin{array}{l}\text { A.S. } \\
\text { D.S. } \\
\text { S.G. }\end{array}$ & $\begin{array}{l}.0015 \\
.0015 \\
.0041\end{array}$ & $\begin{array}{l}.0012 \\
.0017 \\
.0020\end{array}$ \\
\hline 72 Deg & $\begin{array}{l}\text { A.S. } \\
\text { D.S. } \\
\text { S.G. }\end{array}$ & $\begin{array}{l}.038 \\
.12 \\
.13\end{array}$ & $\begin{array}{l}* \\
* \\
*\end{array}$ \\
\hline
\end{tabular}

*Determinant thresholds were not obtained for the highest luminance that was available. The appropriate color response was never given three times in succession.

\section{RESULTS}

\section{Thresholds}

Chromatic thresholds obtained in the two adaptation conditions were compared. These thresholds are presented in Table 1.

The significance of differences was tested separately for each $S$ using a $t$ for paired observations. For both the 510 - and 580-nm stimuli, all Ss showed a higher threshold after dark adaptation, supporting the rod interference hypothesis. All differences were significant $(p<.05)$ except for the 580-nm stimulus for S D.S. The most consistent effect occurred to the 510-nm stimulus, to which rods are most sensitive. If rods are interfering with peripheral color vision, they should not interfere with the $670-\mathrm{nm}$ stimulus. This lack of interference would be expected given the data of Wald (1945), which showed that dark-adapted cones were, if anything, more sensitive to $670 \mathrm{~nm}$ than dark-adapted rods at $8 \mathrm{deg}$. In fact, consistent with the rod interference hypothesis, no significant differences were found at $8 \mathrm{deg}$ for the $670-\mathrm{nm}$ stimulus. If some other factor, such as lowering the criterion for reporting color, influenced the differences found for 510- and 580-nm stimuli, a difference should also be found for the $670-\mathrm{nm}$ stimulus. The lack of a difference for $670 \mathrm{~nm}$ is a further indication that the effects are due to rods. No significant differences were found for the 470-nm stimulus. An interpretation of this lack of a difference will be discussed later.

Evidence for rod interference also occurred at $72 \mathrm{deg}$. Under conditions of dark adaptation, "determinant" color thresholds could not be obtained with the intensity available, i.e., Ss never reported the appropriate color three times in succession. Even at the brightest settings, appropriate color responses never exceeded $50 \%$, as can be seen in Table 2 . After light adaptation, however, determinant color thresholds were obtained. Only S S.G. failed to show a threshold under this condition for the $580-\mathrm{nm}$ stimulus. The cause of this deviance is not known.

The evidence does support the idea that rods interfere with color vision in the periphery. But could this interference come from the rods adding a signal which can be interpreted by the color processing system as a signal for hue? Color responses throughout the photochromatic interval in the dark-adaptation condition were recorded. The number of responses of each response category were summed across Ss and divided by 27 to yield a percentage of responses. Except for values well below achromatic threshold, stimuli at each luminance value of the ascending series were presented nine times to each of three Ss, yielding a total of 27 presentations. Above chromatic threshold, presentation was stopped, but it was assumed that Ss would have continued with the same response. The results are presented in Table 2.

Table 2

\begin{tabular}{|c|c|c|c|c|c|c|c|}
\hline \multirow[b]{2}{*}{ WI $\dagger$} & \multirow{2}{*}{$\begin{array}{l}\text { Lumi- } \\
\text { nance* }\end{array}$} & \multicolumn{5}{|c|}{ Mean Percent of Response } & \multirow[b]{2}{*}{$\mathbf{N}$} \\
\hline & & B & $\mathbf{G}$ & $\mathrm{Y}$ & $\mathbf{R}$ & Ac & \\
\hline \multicolumn{8}{|c|}{ 8-Deg Dark Adapted: 470-nm Stimulus } \\
\hline 0 & .00001 & 11 & $\mathbf{0}$ & 0 & 0 & 37 & 52 \\
\hline 1 & .00002 & 26 & 4 & 0 & 4 & 41 & 25 \\
\hline 2 & .00004 & 22 & 0 & 0 & 0 & 74 & 4 \\
\hline 3 & .00008 & 33 & 4 & 4 & 0 & 59 & 0 \\
\hline 4 & .00016 & 15 & 0 & 4 & 0 & 81 & 0 \\
\hline 5 & .00032 & 22 & 0 & 0 & 0 & 78 & 0 \\
\hline 6 & .00064 & 30 & 4 & 7 & 0 & 59 & 0 \\
\hline 7 & .0012 & 22 & 4 & 4 & 0 & 70 & 0 \\
\hline 8 & .0024 & 22 & 4 & 0 & 0 & 74 & 0 \\
\hline 9 & .0048 & 41 & 4 & 0 & 0 & 55 & 0 \\
\hline 10 & .0096 & 55 & 18 & 0 & 0 & 27 & 0 \\
\hline 11 & .019 & 78 & 11 & 0 & 0 & 11 & 0 \\
\hline 12 & .038 & 81 & 11 & 0 & 0 & 8 & 0 \\
\hline 13 & .076 & 85 & 7 & 0 & 0 & 8 & 0 \\
\hline 14 & .152 & 81 & 11 & 0 & 0 & 8 & 0 \\
\hline 15 & .304 & 96 & 0 & 0 & 0 & 4 & 0 \\
\hline 16 & .608 & 92 & 8 & 0 & 0 & 0 & 0 \\
\hline 17 & 1.22 & 100 & 0 & 0 & 0 & 0 & 0 \\
\hline \multicolumn{8}{|c|}{ 8-Deg Dark Adapted: 510-nm Stimulus } \\
\hline 0 & .00003 & 11 & 0 & 0 & 0 & 51 & 38 \\
\hline 1 & .00006 & 41 & 0 & 0 & 0 & 59 & 0 \\
\hline 2 & .00012 & 26 & 0 & 0 & 0 & 74 & 0 \\
\hline 3 & .00024 & 33 & 0 & 0 & 0 & 67 & 0 \\
\hline
\end{tabular}


Table 2 (Continued)

\begin{tabular}{|c|c|c|c|c|c|c|}
\hline \multirow[b]{2}{*}{ WI $\dagger$} & \multirow{2}{*}{$\begin{array}{l}\text { Lumi- } \\
\text { nance* }\end{array}$} & \multicolumn{5}{|c|}{ Mean Percent of Response } \\
\hline & & B & G & $\mathrm{Y}$ & $\mathbf{R}$ & Ac \\
\hline 4 & .00048 & 30 & 0 & 0 & 4 & 66 \\
\hline 5 & .00096 & 26 & 0 & 0 & 0 & 74 \\
\hline 6 & .0019 & 33 & 0 & 4 & 0 & 63 \\
\hline 7 & .0038 & 18 & 8 & 4 & 0 & 70 \\
\hline 8 & .0076 & 30 & 4 & 0 & 0 & 66 \\
\hline 9 & .015 & 22 & 18 & 0 & 0 & 60 \\
\hline 10 & .030 & 22 & 56 & 0 & 0 & 22 \\
\hline 11 & .060 & 4 & 70 & 0 & 0 & 26 \\
\hline 12 & .122 & 11 & 85 & 0 & 0 & 4 \\
\hline 13 & .244 & 7 & 89 & 0 & 0 & 4 \\
\hline 14 & .488 & 4 & 92 & 0 & 0 & 4 \\
\hline 15 & .976 & 4 & 96 & 0 & 0 & 0 \\
\hline \multirow[t]{2}{*}{16} & 1.95 & 0 & 100 & 0 & 0 & 0 \\
\hline & \multicolumn{6}{|c|}{ 8-Deg Dark Adapted: 580-nm Stimulus } \\
\hline 0 & .00014 & 7 & 0 & 0 & 0 & 51 \\
\hline 1 & .00028 & 18 & 0 & 4 & $\mathbf{0}$ & 74 \\
\hline 2 & .00056 & 18 & 4 & 0 & 0 & 78 \\
\hline 3 & .0011 & 26 & 0 & 4 & 0 & 70 \\
\hline 4 & .0022 & 18 & 0 & 12 & 4 & 66 \\
\hline 5 & .0044 & 7 & 0 & 15 & 4 & 74 \\
\hline 6 & .0088 & 4 & 0 & 18 & 4 & 74 \\
\hline 7 & .018 & 0 & 0 & 33 & 4 & 63 \\
\hline 8 & .036 & 0 & 4 & 59 & 11 & 26 \\
\hline 9 & .072 & 0 & 4 & 81 & 0 & 15 \\
\hline 10 & .144 & 0 & 11 & 89 & 0 & 0 \\
\hline 11 & .288 & 0 & 15 & 85 & 0 & 0 \\
\hline 12 & .576 & 0 & 4 & 96 & 0 & 0 \\
\hline 13 & 1.15 & 0 & 4 & 96 & 0 & 0 \\
\hline \multirow[t]{2}{*}{14} & 2.30 & 0 & 0 & 100 & 0 & 0 \\
\hline & \multicolumn{6}{|c|}{ 8-Deg Dark Adapted: 670-nm Stimulus } \\
\hline 0 & .0017 & 0 & 0 & 0 & 64 & 11 \\
\hline 1 & .0034 & 0 & 0 & 0 & 96 & 4 \\
\hline \multirow[t]{2}{*}{2} & .0068 & 0 & 0 & 0 & 100 & 0 \\
\hline & \multicolumn{6}{|c|}{ 8-Deg Dark Adapted: Achromatic } \\
\hline 0 & .0083 & 11 & 0 & 0 & 0 & 70 \\
\hline 1 & .0017 & 33 & 4 & 0 & 0 & 63 \\
\hline 2 & .0034 & 37 & 0 & 0 & 0 & 63 \\
\hline 3 & .0068 & 33 & 0 & 0 & 0 & 67 \\
\hline 4 & .014 & 30 & 0 & 0 & 0 & 70 \\
\hline 5 & .028 & 11 & 0 & 4 & 0 & 85 \\
\hline 6 & .056 & 11 & 0 & 8 & 0 & 81 \\
\hline 7 & .11 & 8 & 0 & 0 & 0 & 92 \\
\hline 8 & .22 & 4 & 0 & 15 & 0 & 81 \\
\hline 9 & .44 & 0 & 0 & 19 & 0 & 81 \\
\hline 10 & .88 & 0 & 8 & 37 & 0 & 55 \\
\hline 11 & 1.76 & 0 & 8 & 41 & 0 & 51 \\
\hline 12 & 3.52 & 0 & 15 & 51 & 0 & 26 \\
\hline 13 & 7.00 & 15 & 44 & 0 & 0 & 11 \\
\hline 14 & 14.00 & 0 & 11 & 46 & 0 & 11 \\
\hline 15 & 28.00 & 0 & 7 & 30 & 0 & 0 \\
\hline
\end{tabular}

72-Deg Dark Adapted: 470-nm Stimulus

$\begin{array}{lrrrrr}.00002 & 11 & 0 & 0 & 4 & 37 \\ .00004 & 4 & 0 & 0 & 11 & 70 \\ .00008 & 18 & 0 & 0 & 8 & 74 \\ .00016 & 22 & 0 & 0 & 4 & 74 \\ .00032 & 11 & 0 & 0 & 4 & 85 \\ .00064 & 18 & 0 & 0 & 4 & 78 \\ .0012 & 22 & 4 & 0 & 0 & 74 \\ .0024 & 11 & 4 & 0 & 0 & 85 \\ .0048 & 29 & 0 & 4 & 0 & 67 \\ .0096 & 22 & 0 & 11 & 0 & 67 \\ .019 & 11 & 4 & 8 & 0 & 77 \\ .038 & 8 & 8 & 4 & 0 & 80 \\ .076 & 8 & 11 & 4 & 0 & 77 \\ .152 & 19 & 11 & 8 & 0 & 62\end{array}$

Table 2 (Continued)

\begin{tabular}{|c|c|c|c|c|c|c|c|}
\hline \multirow[b]{2}{*}{ WI $\dagger$} & \multirow{2}{*}{$\begin{array}{l}\text { Lumi- } \\
\text { nance* }\end{array}$} & \multicolumn{5}{|c|}{ Mean Percent of Response } & \multirow[b]{2}{*}{$\mathbf{N}$} \\
\hline & & B & $\mathbf{G}$ & $\mathbf{Y}$ & $\mathbf{R}$ & Ac & \\
\hline 14 & .304 & 26 & 15 & 4 & 0 & 55 & 0 \\
\hline 15 & .608 & 37 & 11 & 0 & 0 & 52 & 0 \\
\hline 16 & 1.22 & 37 & 11 & 8 & 0 & 44 & 0 \\
\hline \multicolumn{8}{|c|}{ 72-Deg Dark Adapted: 510-nm Stimulus } \\
\hline 0 & .00006 & 7 & 0 & 0 & 0 & 44 & 49 \\
\hline 1 & .00012 & 11 & $\mathbf{0}$ & 0 & 7 & 74 & 8 \\
\hline 2 & .00024 & 15 & 0 & 0 & 4 & 77 & 4 \\
\hline 3 & .00048 & 19 & 0 & 0 & 4 & 77 & 0 \\
\hline 4 & .00096 & 11 & 0 & 0 & 0 & 89 & 0 \\
\hline 5 & .0019 & 11 & 0 & 0 & 0 & 89 & 0 \\
\hline 6 & .0038 & 11 & 0 & 0 & 0 & 89 & 0 \\
\hline 7 & .0076 & 11 & 0 & 4 & 0 & 85 & 0 \\
\hline 8 & .015 & 11 & 0 & 4 & 0 & 85 & 0 \\
\hline 9 & .030 & 19 & 0 & 4 & 0 & 77 & 0 \\
\hline 10 & .060 & 15 & 4 & 15 & 0 & 66 & 0 \\
\hline 11 & .122 & 15 & 4 & 18 & 0 & 63 & 0 \\
\hline 12 & .244 & 19 & 11 & 26 & 0 & 44 & 0 \\
\hline 13 & .488 & 8 & 26 & 22 & 0 & 44 & 0 \\
\hline 14 & .976 & 15 & 18 & 18 & 0 & 49 & 0 \\
\hline 15 & 1.95 & 23 & 19 & 18 & 0 & 101 & 0 \\
\hline 16 & 3.90 & 15 & 36 & 8 & 0 & 41 & 0 \\
\hline 17 & 7.80 & 15 & 33 & 8 & 0 & 44 & 0 \\
\hline \multicolumn{8}{|c|}{ 72-Deg Dark Adapted: 580-nm Stimulus } \\
\hline 0 & .00056 & 11 & 0 & 0 & 0 & 37 & 52 \\
\hline 1 & .0011 & 15 & 0 & 0 & 0 & 81 & 4 \\
\hline 2 & .0022 & 15 & 0 & 0 & 0 & 85 & 0 \\
\hline 3 & .0044 & 4 & $\mathbf{0}$ & 0 & 0 & 96 & 0 \\
\hline 4 & .0088 & 15 & 0 & 0 & 0 & 85 & 0 \\
\hline 5 & .018 & 7 & 0 & 0 & 0 & 93 & 0 \\
\hline 6 & .036 & 4 & 0 & 0 & 0 & 96 & 0 \\
\hline 7 & .072 & 4 & 9 & 7 & 0 & 85 & 105 \\
\hline 8 & .144 & 7 & 9 & 4 & 0 & 85 & 105 \\
\hline 9 & .288 & 7 & 0 & 4 & 0 & 89 & 0 \\
\hline 10 & .576 & 4 & 0 & 26 & 0 & 70 & 0 \\
\hline 11 & 1.15 & 4 & 11 & 30 & 0 & 55 & 0 \\
\hline 12 & 2.30 & 4 & 22 & 41 & 0 & 33 & 0 \\
\hline 13 & 4.60 & 8 & 30 & 36 & 0 & 26 & 0 \\
\hline 14 & 9.20 & 4 & 30 & 48 & 0 & 18 & 0 \\
\hline 15 & 18.40 & 8 & 26 & 36 & 0 & 30 & 0 \\
\hline
\end{tabular}

72-Deg Dark Adapted: 670-nm Stimulus

$\begin{array}{lrlrrrr}.014 & 4 & 0 & 0 & 4 & 66 & 26 \\ .028 & 15 & 0 & 0 & 8 & 77 & 0 \\ .056 & 8 & 0 & 0 & 11 & 81 & 0 \\ .11 & 4 & 0 & 4 & 11 & 81 & 0 \\ .22 & 4 & 0 & 11 & 11 & 74 & 0 \\ .44 & 0 & 0 & 41 & 15 & 44 & 0 \\ .88 & 0 & 0 & 55 & 15 & 30 & 0\end{array}$

72-Deg Dark Adapted: Achromatic

\begin{tabular}{rrlrlllll} 
& 0 & .0034 & 22 & 0 & 0 & 4 & 37 & 37 \\
48 & 1 & .0068 & 22 & 0 & 0 & 0 & 78 & 0 \\
15 & 2 & .014 & 22 & 0 & 0 & 0 & 78 & 0 \\
0 & 3 & .028 & 11 & 0 & 0 & 0 & 89 & 0 \\
0 & 5 & .056 & 15 & 0 & 0 & 0 & 85 & 0 \\
0 & 6 & .11 & 11 & 0 & 7 & 4 & 78 & 0 \\
0 & 7 & .22 & 4 & 0 & 4 & 4 & 88 & 0 \\
0 & 8 & .84 & 0 & 0 & 0 & 0 & 96 & 0 \\
0 & 9 & 1.76 & 7 & 0 & 0 & 0 & 89 & 0 \\
0 & 10 & 3.52 & 11 & 0 & 7 & 0 & 77 & 0 \\
0 & 11 & 7.00 & 11 & 7 & 7 & 0 & 70 & 0 \\
0 & 12 & 14.00 & 11 & 11 & 7 & 0 & 70 & 0 \\
0 & 13 & 28.00 & 11 & 15 & 7 & 0 & 62 & 0 \\
0 & 13 & 7 & 15 & 11 & 0 & 37 & 0 \\
\hline
\end{tabular}

*fL photopic †Wedge increments above threshold 
The percentage of responses is given for each luminance value in the ascending series, beginning with the luminance level closest to achromatic threshold. It should be noted that for the achromatic stimuli, percentages do not always add to 100 at high luminance levels. This is because presentation of the achromatic stimulus was not always continued to the highest luminance.

At $8 \mathrm{deg}$, there was a tendency for blue to be reported right after the achromatic threshold for all stimuli except $670 \mathrm{~nm}$. This blue should be attributed to rods because it occurs to three widely separated wavelengths. It is also reported well below light-adapted chromatic threshold at a luminance where rods are more sensitive than cones. Krauskopf and Srebro (1965) have found that "small, brief, dim flashes" of monochromatic light in the fovea can produce inappropriate hue sensations. However, these sensations were not uniquely blue. Also, it is not clear that their stimuli were presented anywhere near the luminance of achromatic threshold at $72 \mathrm{deg}$. For these reasons, the Krauskopf and Srebro results would not best account for the present findings. The occurrence of this blue explains why there were no significant differences between light- and dark-adapted thresholds for the $470-\mathrm{nm}$ stimulus at $8 \mathrm{deg}$. This blue response caused high variability for the $470-\mathrm{nm}$ threshold obtained at both 8 and $72 \mathrm{deg}$.

Similar results were obtained at $72 \mathrm{deg}$. Reports of blue occurred near achromatic threshold for all four stimuli. At both 8 and $72 \mathrm{deg}$, similar reports of blue were also obtained near achromatic threshold with the achromatic stimulus.

\section{DISCUSSION}

Increases found in the chromatic threshold under conditions of dark adaptation support the idea that rods do influence color perception.

A hypothesis to explain this finding is that rods contribute an achromatic response which desaturates peripheral colors and so makes them less discriminable. However, the present experiment found that Ss reported lights near the achromatic threshold to be blue. This suggests that the rod response might be treated by the color processing system as a signal for blue as well as white. If so, the rod responses might have the same effect as a signal from the short wavelength cone system. Thus, degraded color vision in the dark-adapted periphery might also be influenced by a change in hue information provided by the color processing system.

Boynton et al (1964) have reported increases in blue and yellow sensations in the dark-adapted periphery when compared to sensations at central fixation. In a color-mixing experiment, Moreland and Cruz (1959) also found increases in the blue component of colors in the dark-adapted periphery. The role that rods played in these results is not clear, because the stimuli used were well above achromatic threshold and probably stimulating both rods and cones. However, the results could have been influenced by rods contributing a blue chromatic component to the color processing system. If rods were contributing blue to the color sensation in these experiments, then the blue component of the colors should change after light adaptation.

\section{REFERENCES}

Barlow, H. B \& Levick, W. R. The Purkinje shift in the cat retina. Journal of Physiology, 1968, 196, 2-3.

Boynton, R. M., Schofer, W., \& Heum, M. E. Hue-wavelength relation measured by color-naming method for three retinal locations. Science, 1964, 146, 666-668.

Cornsweet, T N The staircase method in psychophysics. American Journal of Psychology, 1962, 75, 485-491.

Cornsweet, T. N. Visual perception. New York: Academic Press, 1970. P. 137

Donner, K. O., \& Rushton, W. A. H. Rod-cone interaction in the frog's retina analyzed by the Stiles-Crawford effect and by dark adaptation. Journal of Physiology, 1959, 149, 303-317.

Ferree, F. E., \& Rand, G. Chromatic thresholds of sensation from center to periphery of the retina and their bearing on color theory. Psychological Review, 1919, 26, 16-41.

Gouras, P., \& Link, $K$. Rod and cone interaction in dark-adapted monkey ganglion cells. Journal of Physiology, 1966, 184, 499-510.

Hecht, S., Haig, C., \& Chase, A. Influence of light adaptation on subsequent dark adaptation of the eye. Journal of General Physiology, 1937, 20, 831-850.

Hunt, R. W Light and dark adaptation and the perception of color. Journal of the Optical Society of America, 1952, 42, 190-199.

Krauskopf, J., \& Srebro, R. Spectral sensitivity of color mechanisms: Derivation from fluctuations of color appearance near threshold. Science, 1965, 150, 1477-1479.

Lie, I. Dark adaptation and the photochromatic interval. Documenta Ophthalmologica, 1963, 17, 411-510.

Moreland, J. D., \& Cruz, A. Colour perception with the peripheral retina. Optica Acta, 1959, 59, 117-151.

Roaf, H. E. Colour vision. Physiology Review, 1933, 13, 43-79.

Spillmann, L., \& Conlon, J. E. Photochromatic interval during dark adaptation and as a function of background luminance. Journal of the Optical Society of America, 1972, 62, 182-185.

Spillmann, L., \& Seneff, S. Photochromatic intervals as a function of retinal eccentricity for stimuli of different size. Journal of the Optical Society of America, 1971, 61, 267-270.

Trezona, P. W. Rod participation in the 'blue' mechanism and its effect on colour matching. Vision Research, 1970, 10, 317-332.

Wald, G. Human vision and the spectrum. Science, 1945, 101, 653-658.

(Received for publication July 18, 1973;

final revision received January 15,1974 .) 